\title{
EDGE: Explorer of diffuse emission and gamma-ray burst explosions
}

L. Piro • J. W. den Herder • T. Ohashi - L. Amati •

J. L. Atteia - S. Barthelmy - M. Barbera - D. Barret •

S. Basso - M. Boer - S. Borgani • O. Boyarskiy •

E. Branchini - G. Branduardi-Raymont - M. Briggs •

G. Brunetti • C. Budtz-Jorgensen - D. Burrows •

S. Campana - E. Caroli • G. Chincarini •

F. Christensen - M. Cocchi - A. Comastri

A. Corsi - V. Cotroneo - P. Conconi - L. Colasanti •

G. Cusumano $\cdot$ A. de Rosa $\cdot$ M. Del Santo

S. Ettori • Y. Ezoe • L. Ferrari • M. Feroci •

M. Finger • G. Fishman • R. Fujimoto

M. Galeazzi • A. Galli • F. Gatti • N. Gehrels •

B. Gendre • G. Ghirlanda - G. Ghisellini •

P. Giommi • M. Girardi • L. Guzzo • F. Haardt •

I. Hepburn • W. Hermsen • H. Hoevers

A. Holland • J. in't Zand • Y. Ishisaki •

H. Kawahara - N. Kawai • J. Kaastra • M. Kippen •

P. A. J. de Korte • C. Kouveliotou • A. Kusenko •

C. Labanti - R. Lieu • C. Macculi • K. Makishima •

G. Matt • P. Mazzotta • D. McCammon •

M. Méndez • T. Mineo • S. Mitchell • K. Mitsuda •

S. Molendi • L. Moscardini • R. Mushotzky •

L. Natalucci • F. Nicastro • P. O'Brien • J. Osborne •

F. Paerels • M. Page • S. Paltani - G. Pareschi •

E. Perinati - C. Perola - T. Ponman

A. Rasmussen • M. Roncarelli • P. Rosati •

O. Ruchayskiy • E. Quadrini • I. Sakurai •

R. Salvaterra • S. Sasaki • G. Sato • J. Schaye •

J. Schmitt • S. Sciortino • M. Shaposhnikov •

K. Shinozaki • D. Spiga • Y. Suto • G. Tagliaferri •

T. Takahashi $\cdot$ Y. Takei - Y. Tawara $\cdot$ P. Tozzi •

H. Tsunemi - T. Tsuru • P. Ubertini • E. Ursino •

M. Viel $\cdot$ J. Vink $\cdot$ N. White $\cdot$ R. Willingale $\cdot$

R. Wijers $\cdot$ K. Yoshikawa $\cdot$ N. Yamasaki 
Abstract How structures of various scales formed and evolved from the early Universe up to present time is a fundamental question of astrophysical cosmology. EDGE (Piro et al., 2007) will trace the cosmic history of the baryons from the early generations of massive stars by Gamma-Ray Burst (GRB) explosions, through the period of galaxy cluster formation, down to the very low redshift Universe, when between a third and one half of the baryons are expected to reside in cosmic filaments undergoing gravitational collapse by dark matter (the so-called warm hot

L. Piro $(\bowtie) \cdot$ M. Cocchi $\cdot$ A. Corsi $\cdot$ L. Colasanti $\cdot$ A. de Rosa $\cdot$ M. Del Santo $\cdot$ M. Feroci $\cdot$ A. Galli

B. Gendre $\cdot$ C. Macculi $\cdot$ L. Natalucci $\cdot$ P. Ubertini

INAF, Istituto Astrofisica Spaziale Fisica Cosmica, Rome, Italy

e-mail: luigi.piro@iasf-roma.inaf.it

J. W. den Herder $\cdot$ W. Hermsen $\cdot$ H. Hoevers $\cdot$ J. in’t Zand $\cdot$ J. Kaastra $\cdot$

P. A. J. de Korte · M. Méndez • Y. Takei

SRON, the Netherlands Institute for Space Research,

Sorbonnelaan 2, 3854 CA Utrecht, The Netherlands

T. Ohashi $\cdot$ S. Sasaki $\cdot$ K. Shinozaki

Tokyo Metropolitan University, Tokyo, Japan

L. Amati $\cdot$ E. Caroli $\cdot$ C. Labanti

INAF, Istituto Astrofisica Spaziale Fisica Cosmica, Bologna, Italy

S. Barthelmy $\cdot$ N. Gehrels $\cdot$ R. Mushotzky $\cdot$ G. Sato $\cdot$ N. White

NASA GSFC, Greenbelt, Maryland, USA

G. Cusumano $\cdot$ T. Mineo $\cdot$ E. Perinati

INAF, Istituto Astrofisica Spaziale Fisica Cosmica, Palermo, Italy

\section{J. L. Atteia}

LAT, Observatoire Midi-Pyrénées, Toulouse, France

D. Barret

CESR Centre d'Etude Spatiale des Rayonnements, Toulouse, France

S. Basso $\cdot$ S. Campana $\cdot$ G. Chincarini $\cdot$ V. Cotroneo $\cdot$ P. Conconi $\cdot$ G. Ghirlanda $\cdot$ G. Ghisellini $\cdot$

L. Guzzo • G. Pareschi • D. Spiga • G. Tagliaferri

INAF, Osservatorio Astronomico Brera, Milano, Italy

S. Borgani $\cdot$ M. Girardi $\cdot$ P. Tozzi $\cdot$ M. Viel

INAF, Osservatorio Astronomico, Trieste, Italy

E. Branchini $\cdot$ G. Matt $\cdot$ C. Perola

Università Roma III, Rome, Italy

M. Briggs $\cdot$ R. Lieu

University of Alabama in Huntsville, Hunstville, AL, USA

C. Budtz-Jorgensen · F. Christensen

DNSC/Technical University of Denmark, Copenhagen, Denmark

D. Burrows

Penn State University, University Park, Philadelphia, PA, USA

A. Comastri $\cdot$ S. Ettori $\cdot$ M. Roncarelli

INAF, Osservatorio Astronomico Bologna, Bologna, Italy

yㅡㄹ Springer 
intragalactic medium). In addition EDGE, with its unprecedented capabilities, will provide key results in many important fields. These scientific goals are feasible with a medium class mission using existing technology combined with innovative instrumental and observational capabilities by: (a) observing with fast reaction Gamma-Ray Bursts with a high spectral resolution. This enables the study of their star-forming and host galaxy environments and the use of GRBs as back lights of large scale cosmological structures; (b) observing and surveying extended sources (galaxy clusters, WHIM) with high sensitivity using two wide field of view X-ray telescopes (one with a high angular resolution and the other with a high spectral resolution). The mission concept includes four main instruments: a Wide-field

L. Ferrari $\cdot$ F. Gatti

Istituto Nazionale di Fisica Nucleare, Genova, Italy

M. Finger

University Space Research Association, Huntsville, AL, USA

G. Fishman $\cdot$ C. Kouveliotou $\cdot$ S. Mitchell

Marshall Space Flight Center, Huntsville, AL, USA

M. Galeazzi $\cdot$ E. Ursino

University of Miami, Miami, FL, USA

G. Branduardi-Raymont $\cdot$ I. Hepburn $\cdot$ M. Page

Mullard Space Science Lab/UCL, London, UK

\section{A. Holland}

University of Brunel, London, UK

Y. Ishisaki $\cdot$ I. Sakurai $\cdot$ Y. Tawara

Nagoya University, Nagoya, Japan

N. Kawai

Tokyo Institute of Technology, Tokyo, Japan

M. Kippen

Los Alamos National Laboratory, Los Alamos, NM, USA

Y. Ezoe $\cdot$ R. Fujimoto $\cdot$ K. Mitsuda $\cdot$ T. Takahashi $\cdot$ N. Yamasaki Institute of Space and Aeronautical Science, JAXA, Tokyo, Japan

S. Molendi • E. Quadrini

INAF, Istituto Astrofisica Spaziale e Fisica Cosmica, Milano, Italy

A. Rasmussen

KIPAC/Standord, Palo Alto, California, USA

H. Kawahara $\cdot$ Y. Suto $\cdot$ K. Yoshikawa

University of Tokyo, Tokyo, Japan

F. Paerels

Columbia University, New York, NY, USA

J. Schaye

University of Leiden, Leiden, The Netherlands 
Spectrometer $(0.1-2.2 \mathrm{eV})$ with excellent energy resolution $(3 \mathrm{eV}$ at $0.6 \mathrm{keV})$, a Wide-Field Imager $(0.3-6 \mathrm{keV})$ with high angular resolution $(\mathrm{HPD}=15$ ") constant over the full 1.4 degree field of view, and a Wide Field Monitor $(8-200 \mathrm{keV})$ with a FOV of $1 / 4$ of the sky, which will trigger the fast repointing to the GRB. Extension of its energy response up to $1 \mathrm{MeV}$ will be achieved with a GRB detector with no imaging capability. This mission is proposed to ESA as part of the Cosmic Vision call. We will outline the science drivers and describe in more detail the payload of this mission.

\author{
P. Mazzotta \\ Universitá di Roma Tor Vergata, Rome, Italy \\ T. Ponman \\ University of Birmingham, Birmingham, UK \\ P. Rosati \\ ESO, Garching, Germany \\ M. Boer
}

Observatoire de Haute Provence, Haute Provence, France

P. O’Brien · J. Osborne $\cdot$ R. Willingale

Leicester University, Leicester, UK

R. Wijers

University of Amsterdam, Amsterdam, The Netherlands

O. Boyarskiy

CERN, Geneva, Switzerland

F. Haardt · R. Salvaterra

Universitá di Insubria (Como), Como, Italy

\author{
A. Kusenko \\ UCLA, Los Angeles, CA, USA
}

S. Paltani

Integral Science Data Center, Versoix, Switzerland

O. Ruchayskiy

Institut des Hautes Etudes Scientifiques, Bures-sur-Yvette, France

M. Shaposhnikov

Ecole Polytechnique Fédérale de Lausanne, Lausanne, Switzerland

J. Schmitt

University of Hamburg, Hamburg, FRG

T. Tsuru

Kyoto University, Kyoto, Japan

J. Vink

University of Utrecht, Utrecht, The Netherlands

P. Giommi

ASI Data Center, Rome, Italy

를 Springer 
Keywords X-rays · Cosmology C Clusters · Gamma-ray bursts ·

Warm-hot intergalactic medium $\cdot$ Missions

\section{Introduction}

One of the fundamental issues in astrophysical cosmology is to understand the formation and evolution of structures on various scales from the early Universe up to present time. The Explorer of Diffuse Emission and Gamma-ray burst Explosions (EDGE) will measure three tracers of cosmic structures:

\subsection{Cosmic filaments}

- $\quad$ Detect the largest reservoir of baryons from $\mathrm{z} \sim 1$ to the present time, predicted to reside in the Warm-Hot Intergalactic Medium (WHIM) by measuring densities down to $10^{-5} \mathrm{~cm}^{-3}$ ( $\sim 30$ times less than currently probed within clusters of galaxies)

- Place constraints on the interplay between unconfined baryons and star formation

\subsection{Clusters of galaxies}

- Trace the evolution and physics of clusters from their formation epoch $(\mathrm{z}>1)$

- Measure the thermodynamical and chemical properties of a representative sample out to the virial radius, a fundamental step to qualify clusters as cosmological probes and for constraining their evolution through the link with the WHIM

\subsection{Gamma-Ray Bursts}

- Study the evolution of massive star formation using Gamma-Ray Bursts (GRBs) to trace their explosions back to the early epochs of the Universe $(z>6)$

K. Makishima

Tokyo University of Science, Tokyo, Japan

D. McCammon

University of Wisconsin, Madison, WI, USA

H. Tsunemi

Osaka University, Osaka, Japan

G. Brunetti

INAF-IRA Bologna, Bologna, Italy

L. Moscardini

Universita’ di Bologna, Bologna, Italy

M. Barbera

Dipartimento Scienze Fisiche e Astronomiche, Universita' di Palermo, Palermo, Italy

F. Nicastro

INAF, Osservatorio Astronomico Roma, Rome, Italy

S. Sciortino

INAF, Osservatorio Astronomico Palermo, Palermo, Italy 
- Measure the metals in the host galaxies of GRBs and the explosive enrichment in their close environs out to $\mathrm{z}>6$

Accomplishing a survey and characterization of these components of the baryonic Universe requires high resolution soft X-ray spectroscopy and imaging over a wide field of view, with extremely low background and the ability to rapidly point at bright GRB afterglows.

The proposed EDGE mission will provide direct observational data on these topics. Currently no other mission under study will address these issues directly. In addition EDGE, with its unprecedented observational capabilities, will provide key results for a number of additional science issues including: the study of feedback mechanisms into the interstellar medium (supernova remnants, galaxy/active galactic nuclei outflows), constraints on the dark matter and dark energy content of the Universe (through clusters and GRBs), equation of state of the densest matter (neutron stars), GRB physics, upper limits on light dark matter particles, accurate measurement of the geometry of space-time by measuring X-ray afterglows of black hole mergers detected through gravitational waves, active galactic nuclei and stellar population surveys, and solar system physics.

\section{Science goals}

EDGE will be able to study the role of the baryons in the Universe from the early epochs, though GRB explosions, via the period of cluster formation, down to very low redshifts. The unique way in which EDGE will contribute to this study is schematically illustrated in Fig. 1. Due to the formation of the first stars, protogalaxies, and massive black holes

\section{Evolution of the Universe}

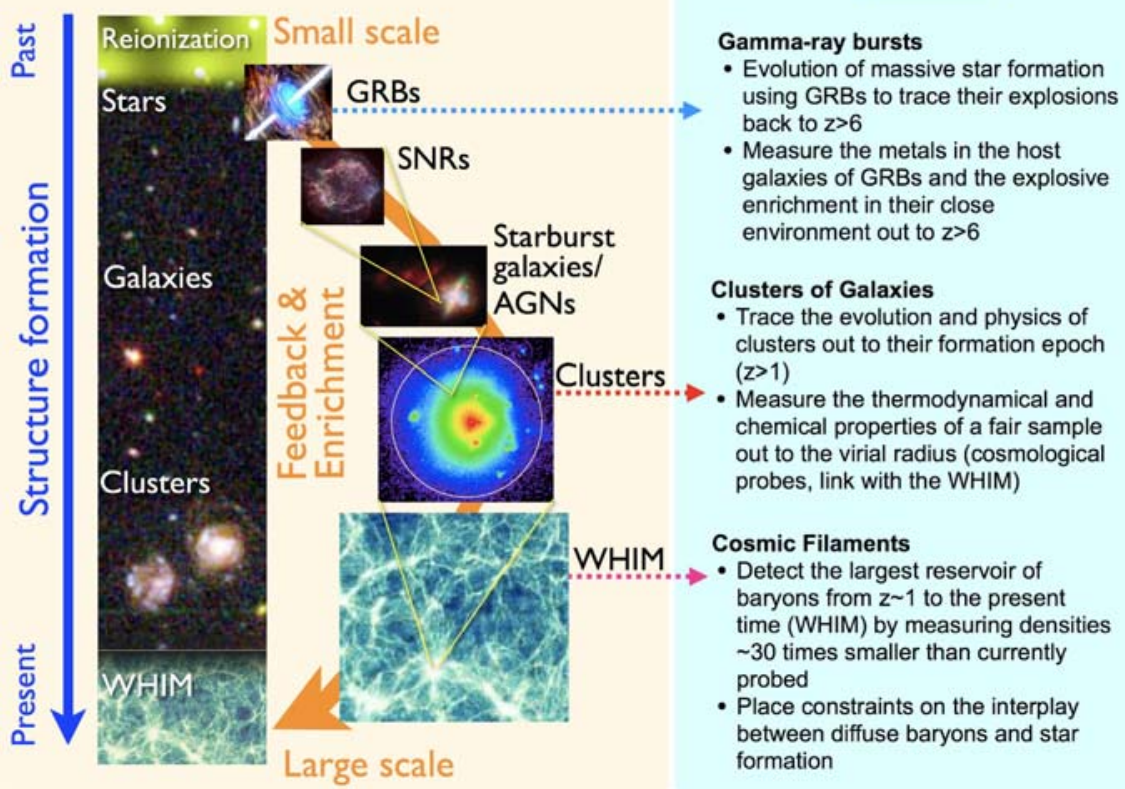

Fig. 1 History of structure formation in the Universe (left) and key EDGE capabilities (right) 
the Universe reionizes. As cosmic structures take shape, gravitational collapse of Dark Matter halos creates the seed regions where star formation occurs; subsequently galaxies form and merge into groups and clusters through a hierarchical build-up process. EDGE traces this evolution by observing the death of massive stars that explode as GRBs back to the first generations in the dark Universe. The associated supernovae inject nucleosynthesis products and energy into their environment. EDGE will detect this first metal enrichment through X-ray absorption spectroscopy of bright GRB afterglows.

Clusters of galaxies are the last and most massive structures to collapse. Besides containing a significant portion of the total mass, the outskirt regions of clusters show the most visible signs of the hierarchical formation of cosmic structures, of the accretion shocks and turbulence injection, as well as of the metal enrichment from relatively recent star formation. EDGE will conduct high sensitivity imaging and high-resolution spectroscopy out to the virial radius, thus performing a detailed thermo- and chemo-dynamical study of diffuse baryons inside and around clusters. Moreover, EDGE will carry out surveys that will detect the formation of the first cluster-sized objects thereby placing tight constraints on the values of cosmological parameters by tracing the growth-rate of cosmic density fluctuations.

As we approach the current epoch, between a third and one half of the baryons are expected to reside in the WHIM [2], which is currently undergoing gravitational collapse. The bulk of this medium remains still undetected. EDGE will observe the WHIM and measure its properties via absorption and emission in atomic transitions of highly ionized metals. These observations close the baryon budget at $z=0$ by constraining their density. In addition, EDGE will shed light on the star formation history from the imprint by the energy and metal injection left on the WHIM.

The unique capability of EDGE on diffuse structures is visualized in Fig. 2 through the phase diagram of the baryons at $z=0$ as predicted by a cosmological

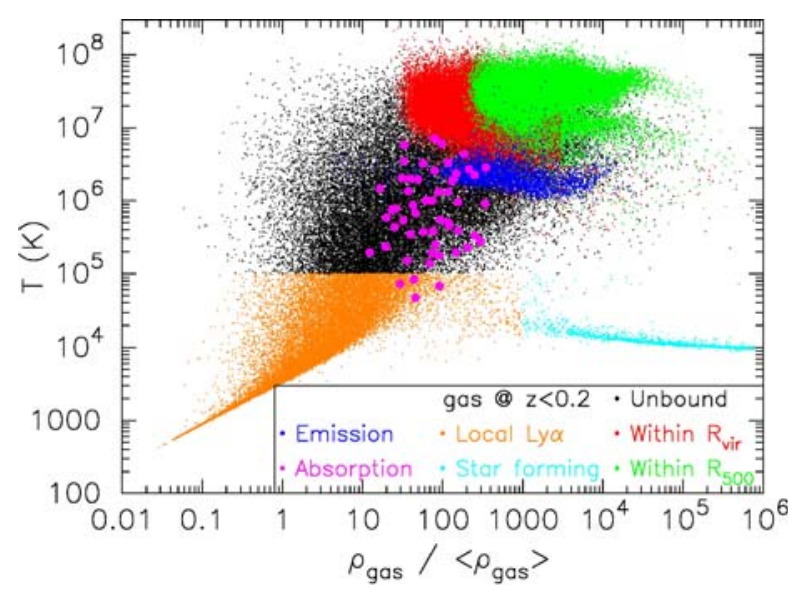

Fig. 2 The EDGE contribution to the sampling of the phase diagram of cosmic baryons (from the hydrodynamical simulation of Borgani et al. [17] at $z \sim 0$ ). EDGE will extend the current observations of the central regions of galaxy clusters (green and top right) out to their outskirts (red, top center) and to the dense part of the WHIM (blue, just below the part filled by clusters), down to the regions with lower density (overplotted purple dots). The star forming region is the sharp line at higher densities and lower temperatures $\left(T<10^{5}\right)$ and the Lyman-forest is the orange area at the bottom left (see online paper for color plot) 
hydrodynamical simulation. For gas with the lowest density in the Intergalactic Medium (IGM) to the densest gas in star-forming galaxies, we plot gas temperature versus normalized density $(\rho /<\rho>)$. Current observations are effectively limited to the cooler parts of this diagram $\left(\mathrm{T}<10^{5} \mathrm{~K}\right)$ and to the hotter and denser parts (clusters of galaxies $\mathrm{T}>10^{7} \mathrm{~K}, \rho /<\rho>>300$ ). EDGE will provide observational access into yet unexplored regions, where most of the baryons resides: the WHIM filaments and the outskirts of cluster of galaxies.

\subsection{Warm-hot intergalactic medium}

Most of our knowledge about the formation of cosmic structures on various scales is based on observations of baryons locked in relatively dense gas accumulated in stars, galaxies, and clusters of galaxies. Surprisingly, current state-of-the-art observations only account for about $30 \%$ to $50 \%$ of the baryons at redshift $\mathrm{z} \sim 0$, while the rest of them is thought to be in diffuse, highly ionized, intergalactic gas that permeates the cosmic web: the so-called Warm-Hot Intergalactic Medium. The WHIM retains key information on the history of gravitational collapse and heating at the accretion shocks, and on the time-dependent kinetic energy injection from galactic winds and AGN jets. The elemental abundances of this gas reflect the history of metal enrichment from the very early times on.

This gas is extremely hard to detect: $\mathrm{H}$ and $\mathrm{He}$ are fully ionized, but the thermal continuum emission is much too faint to be detectable against overwhelming backgrounds. The only characteristic radiation from this medium will be in the discrete transitions of highly ionized $\mathrm{C}, \mathrm{N}, \mathrm{O}, \mathrm{Ne}$, and possibly Fe: to detect the lines associated to such transitions requires high resolution $\mathrm{X}$-ray absorption and emission line spectroscopy and imaging. The predicted fluxes are below the reach of current instrumentation; a single possible detection of intervening O VII/VIII absorption towards Mrk 421 with the Low Energy Transmission Grating Spectrometer on Chandra was not confirmed in a deeper spectrum with the Reflection Grating Spectrometer on XMM-Newton [18, 19]. On the other hand, UV observations have provided convincing evidence that metals exist in the diffuse gas at $\mathrm{z} \sim 0$, as revealed by the OVI and CIII absorption lines [20]. However, these lines probe the cooler part of the WHIM with $T=1-510^{5} \mathrm{~K}$, that contain only $\sim 7 \%$ of the missing baryons.

To observe the WHIM absorption lines one needs bright X-ray background sources that should also be common enough to provide a good statistical sample. This excludes rare objects, like the Blazars during their active phase. Bright QSOs are the most natural candidates but have the disadvantage of being nearby. Indeed only 20 QSOs bright enough to detect WHIM lines in a deep (1 Ms) observation with the WFSpectrometer can be found at $\mathrm{z}>0.3$ and their number drops rapidly with z. Deeper probes are to be preferred since they increase the chance of detecting absorption lines and also allow to trace the evolution of the WHIM. In this respect GRBs are the perfect sources since they are both distant and bright. By using GRBs EDGE will provide unique data on the WHIM. This is illustrated in Fig. 3 where a typical emission and absorption spectrum is shown. The selected viewing direction contains two filaments at different redshifts and the emission and absorption lines are indicated. The good spectral resolution is required to separate the true WHIM lines from the dominant Galactic foreground. Absorption measurements are carried out by 

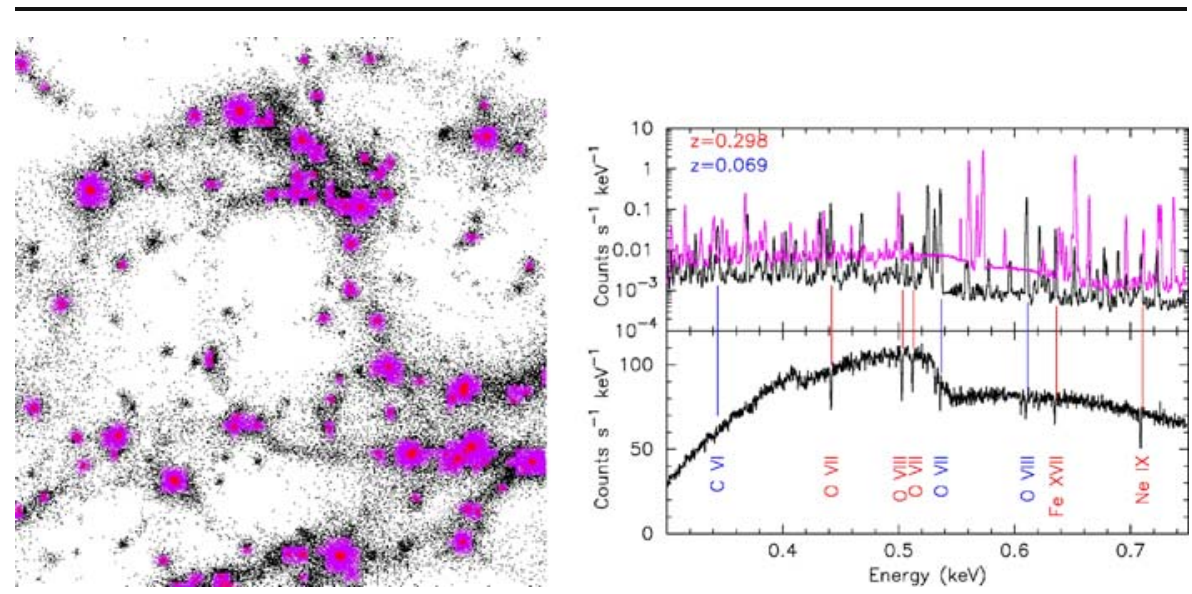

Fig. 3 Left: Sky distribution of O VII emission relative to that of the gas, showing the simulated gas particles (black) and $5 \sigma$ detections of gas with overdensity $\delta>1000$ (red), and with $\delta<1000$ (purple). Area shown is $2.1 \times 2.1 \mathrm{deg}^{2}$ centered on a redshift slice at $z=0.2$ and for a $1 \mathrm{Ms}$ observation. Right: Emission spectrum of a $4 \operatorname{arcmin}^{2}$ area (top) and absorption (bottom) spectrum of the same region of the sky as measured with EDGE. In the top panel the emission of two redshifted components is shown, whereas the emission of the Galactic foreground is shown in light grey and is about a factor 10 more intense. In the bottom panel the spectrum of the same systems is shown but now in absorption using a bright GRB as a beacon

using GRBs as bright back-light sources. Compared to AGNs, GRBs have two advantages. First, they are located at much farther distances, thus allowing to probe filaments back to the era where they formed, around $z=1$. Second, they are transient sources, thus allowing to carry out deep follow up observation of a same filament in emission. We expect to observe about $30 \mathrm{GRBs}$ per year with a sufficient intensity to measure the combined absorption in OVII/VIII with at least $5 \sigma$ corresponding to typically 50 absorption systems (more than one per observation). In addition we plan to observe a $3 \times 3 \mathrm{deg}^{2}$ contiguous area on the sky. The high spectral resolution will thus allow to perform a 3D tomography of the filaments (Fig. 3, left panel). Furthermore we will select a deep image of a few systems observed in absorption, thus enabling the characterization of the WHIM both in emission and absorption. Detailed simulations indicate that, depending on the assumed model, we will detect $18-25 \%$ of the baryons down to overdensities of about 100 . While mere detection of $\mathrm{X}$-ray absorption and emission from highly ionized $\mathrm{C}, \mathrm{N}, \mathrm{O}$, and Fe will reveal the presence of the 'missing baryons', our goal is more ambitious: we plan not just to detect but also to characterize the physical state of the WHIM: its temperature, density, location and metal content which trace the evolution of the medium and its interplay with the history of star formation.

\subsection{Physics and evolution of clusters of galaxies}

Clusters of galaxies are the largest structures to have decoupled from the Hubble flow. While the bulk of the mass in clusters is in the form of dark matter, the dominating baryonic component is a hot tenuous X-ray emitting Intra-Cluster Medium (ICM). All observations of this medium to date have only sampled the tip of the iceberg: only regions within $\mathrm{R}_{500}$, that is $\sim 1.5 \mathrm{Mpc}$, or about $50 \%$ of the virial radius $\left(\mathrm{R}_{200}\right)$ for a 
$10^{15} \mathrm{M}_{\odot}$ object, have been well measured. Thus only a small fraction of the total volume of clusters has been mapped, missing those regions where a sizeable fraction, and for less massive systems in fact most, of the baryons reside and accrete from the field, and where the ICM is heated and enriched by metals. These low surface brightness regions are of course also organically connected to the WHIM. Chandra and XMM-Newton have provided gas distributions for a few massive systems which are already in place by $z \approx 1$, suggesting a formation age that dates back to $z>1.5$. Several fundamental questions are still open: (a) when and how do clusters form? (b) what is the thermodynamic history of the ICM, and what is the role of non-gravitational heating mechanism? (c) how is the ICM polluted by metals? (d) how do the cluster scaling relations take shape?

EDGE will conduct high sensitivity imaging and high-resolution spectroscopy out to the virial radius, thus performing a detailed thermo- and chemo-dynamical study of diffuse baryons inside and around clusters. Moreover, EDGE will carry out surveys that will detect the formation of the first cluster-sized objects, and will allow to address the open questions mentioned above. In addition, the direct measurement of temperature for a large sample of clusters by EDGE will allow to place tight constrains on cosmological parameters, exploiting at the best the potential of clusters of galaxies as tools for precision cosmology [e.g. 6].

This is illustrated in Fig. 4. Thanks to its very low background and stable PSF over the full FoV the WFImager on EDGE will measure surface brightness, temperature and metal abundances out to the virial radius for a sample of clusters. This is approximately a factor 2 larger than current observatories and is, in particular, of importance when using clusters as cosmological probes. In addition, a deep survey, also required to map and characterize the WHIM in emission, will allow us to obtain a sample of clusters out to $z>1$ and to determine for a reasonable subset $(\sim 800$ in 3 years) also the temperature from the X-ray data. For this purpose we expect to use the survey data available in our core program $\left(340 \mathrm{deg}^{2}\right.$ with $50 \mathrm{ks}$ exposure, $11.5 \mathrm{deg}^{2}$ with $1 \mathrm{Ms}$ and $8 \mathrm{deg}^{2}$ with $2 \mathrm{Ms}$ corresponding to a limiting flux of $19,1.5$ and $0.910^{-16} \mathrm{erg} \mathrm{cm}^{-2} \mathrm{~s}^{-1}$ in the $0.5-2 \mathrm{keV}$ band). Note that the confusion
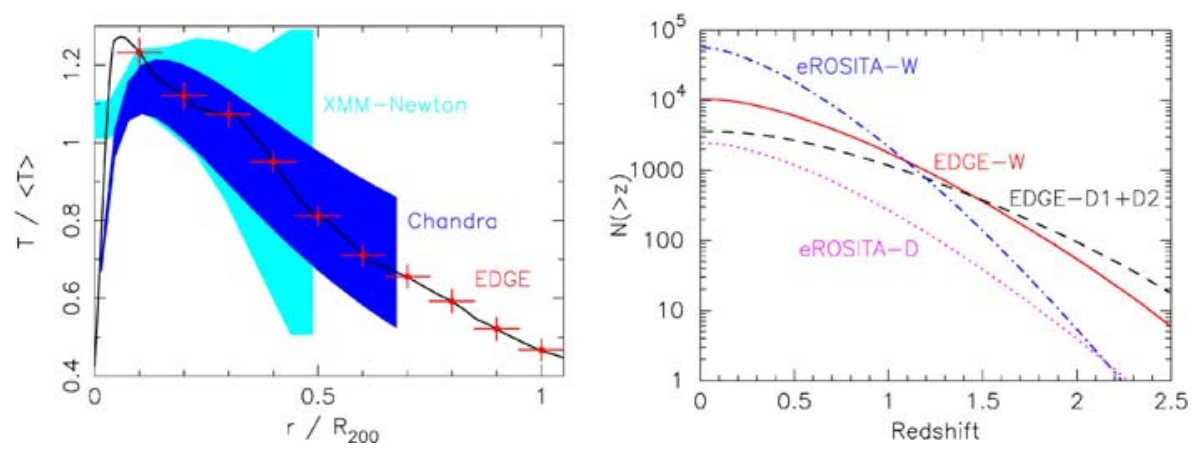

Fig. 4 Left: typical inter cluster medium temperature profiles for current missions and expected EDGE measurements. The model (solid line) is from a hydrodynamical calculation of a massive system $\left(M_{\text {virial }}=\right.$ $2.110^{15} \mathrm{M}_{\odot}$ ) [3]. Right: cumulative redshift distribution of EDGE surveys. These predictions are based on the halo mass function computed for the best-fit WMAP-3yr cosmology and by extrapolating the evolution of the relation between mass and observed X-ray luminosity at $z<0.6$ (e.g. [4, 5]) 
limit is $1.510^{-16}$. This sample will complement the results of future deep surveys in the optical/near-IR and millimeter bands (Sunyaev-Zeldovich effect), sampling large volumes at $\mathrm{z}>1$.

\subsection{From the local to the dark universe with GRBs}

Detailed spectroscopic study of GRB afterglows opens a completely new window on the formation and evolution of the baryons at high redshifts. While currently planned IR/ sub-mm facilities (e.g. JWST, ALMA) will probe the formation and evolution of the earliest massive protogalaxies, EDGE will directly reveal the processes associated with the evolution of the early generation of stars, possibly including the first pop III generation [21], by detecting the GRB heralding the demise of the most massive early generation objects. GRBs (in their long flavor) are indeed associated with SN-like explosions of massive stars. Taking into account SWIFT results and theoretical predictions, we expect to localize and follow up about 20 burst at $z>6$ in 3 years, 7 of which will be characterized by an afterglow fluence $>10^{-6} \mathrm{erg} \mathrm{cm}^{-2}$. This number can be doubled by lowering the nominal trigger threshold of the WFM by about $50 \%$.

GRBs (in their long flavor) show intrinsic (i.e. in situ) X-ray absorption typical of star forming regions. This absorption, which is primarily due to metals, indicates that the column density of metals is $\mathrm{AxN}_{\mathrm{H}}=10^{22} \mathrm{~cm}^{-2}$ [7], where $\mathrm{A}$ is the metallicity relative to solar. It is indeed tantalizing that significant absorption is detected even in the highest redshift burst $(z=6.3)$, indicating that metal enrichment was already vigorous at even earlier epochs. With high resolution spectroscopy, EDGE will see directly the metal enrichment in the environment of the most massive stars. At the highest redshifts we will see the medium- $Z \alpha$-elements ( $\mathrm{Si}$ and upward), and probe for the presence of Fe at these very early times. The exact epoch of first significant Fe enrichment is expected to signal the arrival of supernovae, and therefore probes the prior integral star formation rate.

This is illustrated in Fig. 5 where we show the spectrum of a redshifted GRB $(z=7)$. Clearly the redshift and the column densities for the different edges can be accurately determined.

X-ray spectroscopy, contrary to for instance the optical spectroscopy, provides a direct measurement of the column densities of elements in all physical and chemical states. The ISM in our own Galaxy, observed through bright X-ray binaries [8], has yielded dozens of detections of all abundant elements, in monatomic gaseous, molecular, and solid phases. The strongest absorption lines expected from the neutral or mildly ionized phase are $1 \mathrm{~s}-2 \mathrm{p}$ transitions of C I-III, O I-III, Ne II-IV lines as well as $\mathrm{K}$ and $\mathrm{L}$ edges from the same elements. With EDGE it will be possible to apply this technique to characterize the ISM of GRB host galaxies (Fig. 5 right panel). Expected EWs range from 0.1 to $1 \mathrm{eV}$ for O I or Fe I for column densities down to $10^{21} \mathrm{~cm}^{-2}$. The redshift of each line can be measured with an accuracy of $10^{-3}$, thus enabling kinematic studies of the gas.

The detection of resonant absorption lines from highly ionized metals, detectable only in soft X-rays, will enable the study of the warm-hot phase of the host galaxy inter stellar medium or host galaxy halo (already detected in our own Galaxy). They will be particularly important, in the context of large scale structure evolution, to disclose the presence of hot galactic outflows enriching the IGM well before the 

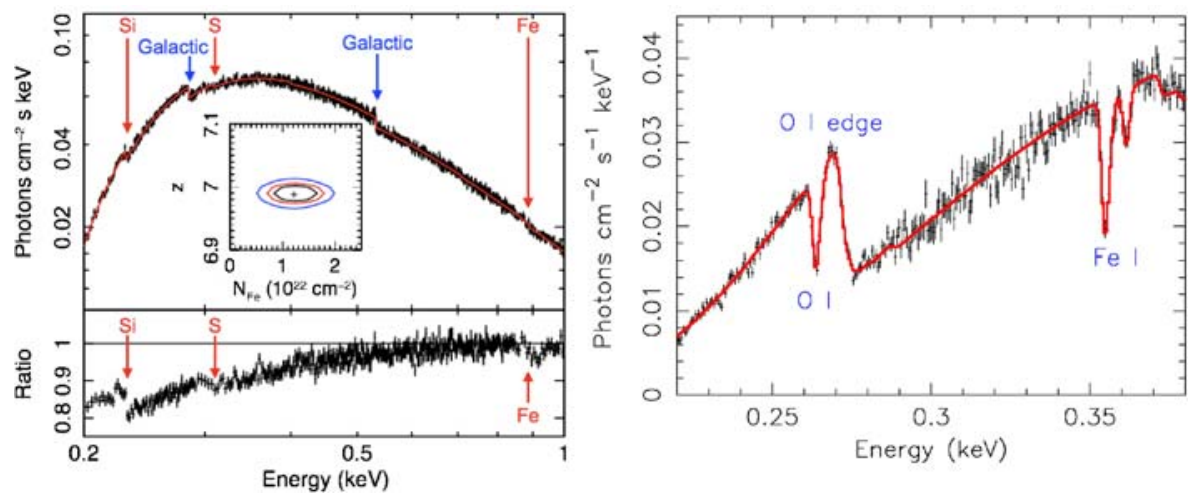

Fig. 5 Left: Spectrum of a $z=7$ GRB afterglow with a fluence of $410^{-6} \mathrm{erg} \mathrm{cm}^{-2}(0.3-10 \mathrm{keV})$, a column density of $\mathrm{N}_{\mathrm{H}}$ of $510^{22} \mathrm{~cm}^{2}$ and an abundance of $1 / 3$ solar. Edges produced by $\mathrm{Si}, \mathrm{S}$, and Fe are clearly detected. Based on these edges the redshift and column densities can be accurately determined $(\Delta z \approx 0.02)$. Right: Spectrum of a GRB at $z=1, \mathrm{~N}_{\mathrm{H}}$ (host galaxy) of $310^{21} \mathrm{~cm}^{-2}, T=210^{4} \mathrm{~K}$, a fluence at $0.3-10 \mathrm{keV}$ of $410^{-6} \mathrm{erg} \mathrm{cm}^{-2}$, integration time $50 \mathrm{ks}$

epoch when WHIM filaments formed. The most promising of such transitions, at high redshifts, are undoubtedly the $\mathrm{K}_{\alpha}$ inner shell bound-bound transitions from mild- to high-ionization Fe (XVII and above).

A very high ionization phase is expected to develop resulting from the ionizing flux produced by the GRB in its close environment. This phase can be easily distinguished from the hot ISM in the host galaxy. Tracking the temporal evolution of absorption features (edges and lines) with EDGE will allow an unprecedented characterization of the circumburst medium (density, metal content and distance from the GRB), which in turn enlighten the origin of the progenitor.

The goal is to gather a sample of about 150 GRB afterglows (in 3 years) with $0.3-10 \mathrm{keV}$ fluences $>10^{-6} \mathrm{erg} \mathrm{cm}^{-2}$. This requires to localize and follow up about 250 GRBs. For this sample we will measure directly the redshift, characterize the star formation, and study the history of metals in both the close GRB environment and its host galaxy up to high-z by X-ray spectroscopy.

\section{Mission}

The measurement of low surface brightness objects and weak emission lines or absorption features is extremely challenging and requires a well balanced combination of instrumental capabilities including:

High resolution $X$-ray spectroscopy with moderate imaging capability

Wide sky monitoring to find GRBs

Fast autonomous pointing towards transient sources

Wide field coverage $\left(\sim 1 \mathrm{deg}^{2}\right)$ with long observations ( Ms)

Employing recent developments in detector and mirror technology it is now possible to perform these measurements. The requirements for the various key science topics are listed in Table 1 where we also indicate the prime instruments (see payload section for details). 
Table 1 Overview of the science requirements

\begin{tabular}{|c|c|c|c|c|c|c|}
\hline Science & $\begin{array}{l}\text { Effective } \\
\text { area }\left[\mathrm{cm}^{2}\right]\end{array}$ & $\begin{array}{l}\text { Energy range } \\
{[\mathrm{keV}]}\end{array}$ & $\begin{array}{l}\text { Ang. } \\
\text { Res. }\end{array}$ & $\begin{array}{l}\text { Field of view } \\
{\left[\operatorname{deg}^{2}\right]}\end{array}$ & $\begin{array}{l}\text { Spectral } \\
\text { resolution }\end{array}$ & Instrument \\
\hline $\begin{array}{l}\text { Missing baryons in } \\
\text { absorption }\end{array}$ & $\begin{array}{l}1000 @ \\
0.5 \mathrm{keV}\end{array}$ & $0.2-1$ & $\mathrm{n} / \mathrm{a}$ & $\mathrm{n} / \mathrm{a}$ & $\begin{array}{l}3 \mathrm{eV} @ \\
0.5 \mathrm{keV}\end{array}$ & WFS+WFM \\
\hline $\begin{array}{l}\text { Missing baryons in } \\
\text { emission }\end{array}$ & $\begin{array}{l}1000 @ \\
0.5 \mathrm{keV}\end{array}$ & $0.2-1$ & $4^{\prime}$ & $0.7 \times 0.7$ & $\begin{array}{l}3 \mathrm{eV} @ \\
0.5 \mathrm{keV}\end{array}$ & WFS+WFI \\
\hline Cluster physics & $\begin{array}{l}500 @ \\
1 \mathrm{keV}\end{array}$ & $0.3-4$ & $15 "$ & $1.4 \varnothing$ & $\begin{array}{l}80 \text { eV@ } \\
1 \mathrm{keV}\end{array}$ & WFI+WFS \\
\hline $\begin{array}{l}\text { Cluster formation and } \\
\text { evolution }\end{array}$ & $\begin{array}{l}500 @ \\
1 \mathrm{keV}\end{array}$ & $0.3-4$ & $15 "$ & $1.4 \varnothing$ & $\begin{array}{l}80 \text { eV@ } \\
1 \mathrm{keV}\end{array}$ & WFI+WFS \\
\hline $\begin{array}{l}\text { From local to Dark Universe } \\
\text { (GRB) }\end{array}$ & $\begin{array}{l}1000 @ \\
0.5 \mathrm{keV}\end{array}$ & $0.2-2$ & $\mathrm{n} / \mathrm{a}$ & $\mathrm{n} / \mathrm{a}$ & $\begin{array}{l}3 \mathrm{eV} @ \\
0.5 \mathrm{keV}\end{array}$ & WFS+WFM \\
\hline
\end{tabular}

\subsection{Launch and orbit}

The required low background dictates a low equatorial earth orbit (LEO). While this has a number of additional advantages (suitability of VEGA launcher, S-band communication), it results in additional constraints on the thermal design due to the vicinity of the earth. Initial analysis has demonstrated that this is feasible, and a low earth orbit is currently also planned for the Japanese NeXT mission [9].

The instruments will be accommodated on a single three-axis stabilized spacecraft which provides all necessary services (Fig. 6). A noteworthy property of EDGE is the fast and autonomous repointing capability. Following the onboard detection of a GRB by the WFMonitor, the satellite will be able to slew quickly and autonomously to its position. The accuracy of the new pointing $\left(4^{\prime}\right)$ is compatible with the sector of the WFSpectrometer which can handle high count rates without loss in spectral resolution. In addition to the usual ground contact during the passage of the satellite over the ground station, a continuous capability to transfer GRB related information

Fig. 6 Satellite in its launch configuration

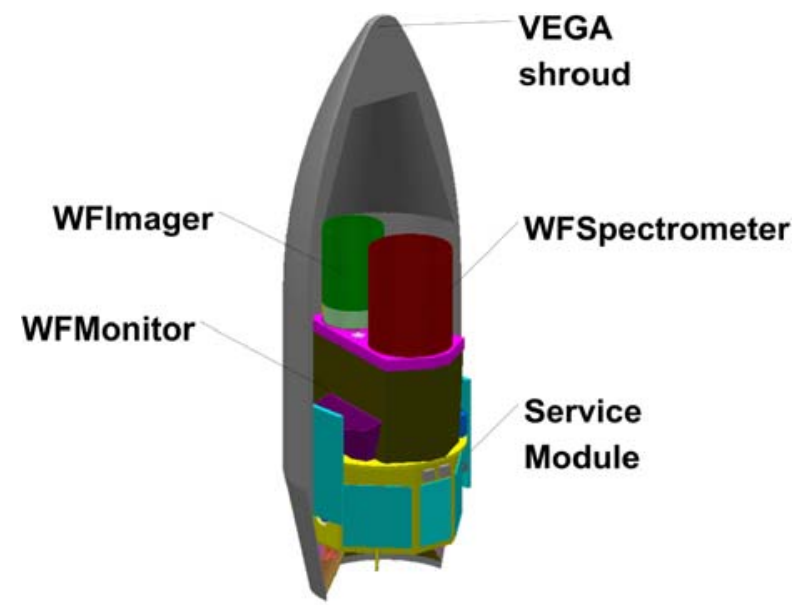


Table 2 Mission characteristics

\begin{tabular}{|c|c|c|}
\hline Parameter & Requirement & Conformance \\
\hline Orbit & $\mathrm{LEO}, \leq 5^{\circ}$ inclination & Compliant \\
\hline Pointing & 3 axis stabilized & better than $2^{\prime}$, knowledge $<1$ " \\
\hline Re-pointing & $60^{\circ}$ in $60 \mathrm{~s}$ & $\begin{array}{l}\text { Within } 60 \mathrm{~s} \text { for } 80 \% \text { of the slews, and } \\
\text { within } 45 \mathrm{~s} \text { for } 50 \% \text { of slews }\end{array}$ \\
\hline Lifetime & 3 years & $\begin{array}{l}5 \text { years, longer lifetime feasible if funding } \\
\text { permits }\end{array}$ \\
\hline Mass & $1932 \mathrm{Kg}$ & Launch capability: $2300 \mathrm{~kg}$ \\
\hline power generation & $3800 \mathrm{~W}$ & $\begin{array}{l}\text { Includes battery charge and peak power } \\
\text { during repointing }\end{array}$ \\
\hline Telemetry & $3.8 \mathrm{Mbps}$ continuous coverage & $\begin{array}{l}\text { Compliant ORBCOMM system for small } \\
\text { volumes of data }\end{array}$ \\
\hline Observing Efficiency & $70 \%$ & Compliant, similar to SWIFT \\
\hline
\end{tabular}

(positions, spectra, etc.) to the ground will be implemented. This system can also be used to upload TOOs to the satellite with a reaction time on the order of minutes (compared to reaction times of hours).

The main satellite requirements are given in Table 2. As indicated, we have selected a nominal duration of 3 years, sufficient to achieve the main science goals of EDGE. The extended lifetime of the mission ( 5 years) can be achieved with a reasonable level of redundancy and reliability, consistent with the envelope of an M-class mission.

\subsection{Observation program}

The observation program includes two parts:

The core program This is $\sim 80 \%$ of the observing time during the first three years. The driving science topics will be addressed in this time. It includes deep observations of (a) the WHIM $(3 \times 3 \mathrm{deg}$ contiguous part of the sky, 3 follow up observations for WHIM filaments detected in absorption) and (b) clusters ( 9 clusters observed for $1 \mathrm{Ms}$ plus a similar observing time for the background), and (c) GRB follow up measurements (240 50 ks observations of GRBs). These data will be made public periodically (once per month). The long observations for the core program will have a multiple use: e.g. the contiguous area for the WHIM will also be a sensitive survey of clusters of galaxies, will enable the study of time variability in various sources etc.

A guest observer (GO) program $\sim 20 \%$ of the observing time which will be assigned following normal ESA guest observer practice.

\subsection{Edge and other facilities}

EDGE is highly complementary to existing and planned X-ray missions. Its high spectral resolution and wide field-of-view, supplemented by the high angular resolution of a more classical X-ray telescope, makes it unique for the detection of weak emission and absorption features in extended sources. This is illustrated in 

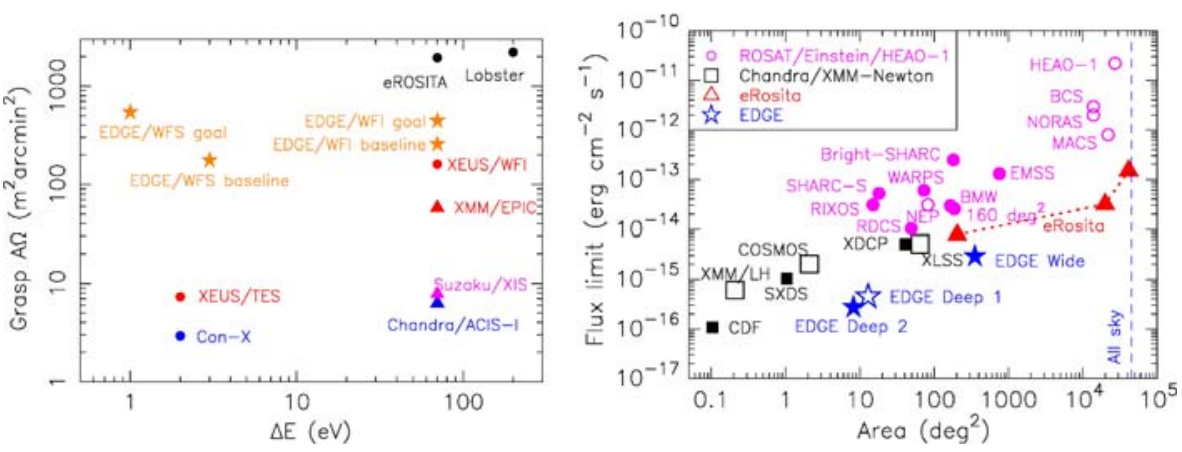

Fig. 7 Left: GRASP and energy resolution @ $0.5 \mathrm{keV}$ for current and planned missions. The GRASP of NeXT is well below Con-X. Right: Solid angles and flux limits of X-ray cluster surveys carried out over the last 2 decades or planned in the future (eROSITA)

Fig. 7 where the grasp (effective area x solid angle) and energy resolution are plotted for current and planned missions. By a grasp which is about two orders of magnitude better than future observatories, in combination with an energy resolution, EDGE will be significantly more sensitive for weak line detection of extended sources than any of these missions.

A second unique feature of EDGE is its ability to acquire deep exposures of a large field-of-view with good and constant spatial resolution, by placing this suite of instruments in a low background orbit. This results in flux limits comparable to, or better than, many performed and planned surveys. This is shown in Fig. 7, where flux limits and sky area of performed surveys and the planned eRosita survey are given. The good PSF over the full WFI FoV, combined with its low instrumental background (due to the LEO) and small $f$ number, will result in sensitive surveys over a large solid angle. At this sensitivity the 15 " instrument PSF is just consistent with the confusion limit.

A third feature of EDGE is the fast repointing of the satellite. This enables for the first time early high spectral resolution observations of bursting sources. It allows us to study the properties of the GRBs and their local environment.

\section{Payload}

A single instrument is not able to provide simultaneously good spectral resolution, good angular resolution, low background, and triggers for fast repointing. Hence, EDGE has a set of four complementary instruments which, together, meet the science goals of the mission:

- The Wide-Field Spectrometer (WFS) which has a cryogenic detector (50 mK) with high spectral resolution and a modest point spread function. The WFS focal length is very short to obtain a large field-of-view for a very small detector. The required modest angular resolution (4') can be provided using light-weight foil optics. 
Table 3 Payload characteristics

\begin{tabular}{|c|c|c|c|c|}
\hline Parameter & WFSpectrometer & WFImager & WFMonitor & GRBDetector \\
\hline Energy resolution & $3 \mathrm{eV} @ 0.5 \mathrm{keV}$ & 70 eV@1keV & 3\%@100keV & 15\%@100keV \\
\hline Energy range $[\mathrm{keV}]$ & $0.1-2.2$ & $0.3-6.0$ & $8-200 \mathrm{keV}$ & $20-3000 \mathrm{keV}$ \\
\hline Angular resolution & $3.7^{\prime}$ & $15 \%$ & 34’ (4'position) & $\mathrm{n} / \mathrm{a}$ \\
\hline Field of View & $0.7 \times 0.7 \mathrm{deg}^{2}$ & $1.5 \operatorname{deg} \varnothing$ & $2.6 \mathrm{sr}$ & $4.4 \mathrm{sr}$ \\
\hline On-axis $A_{\text {effective }}\left[\mathrm{cm}^{2}\right]^{1)}$ & $\begin{array}{l}1163 @ 0.6 \mathrm{keV} \\
499 @ 1.5 \mathrm{keV}\end{array}$ & $\begin{array}{l}580 @ 1 \mathrm{keV} \\
150 @ 3 \mathrm{keV} \\
25 @ 6 \mathrm{keV}\end{array}$ & $1100 @ 100 \mathrm{keV}$ & $1140 @ 600 \mathrm{keV}$ \\
\hline $\operatorname{grasp}(\mathrm{A} \times \Omega)$ & $405 @ 0.6 \mathrm{keV}$ & $\begin{array}{l}750 @ 0.6 \mathrm{keV} \\
1160 @ 1 \mathrm{keV}\end{array}$ & $\mathrm{n} / \mathrm{a}$ & $\mathrm{n} / \mathrm{a}$ \\
\hline Peak countrate & 10.000 & 10.000 & 5000 & 200.000 \\
\hline $\begin{array}{l}\text { Instr. Background @ 1keV } \\
{\left[\text { counts } \mathrm{cm}^{-2} \mathrm{~s}^{-1} \mathrm{keV}^{-1}\right]}\end{array}$ & $510^{-3}$ & $1.110^{-3}$ & & \\
\hline Number of units & 1 & 1 & 2 & 2 \\
\hline Basic mass (excl. margin) & $324 \mathrm{~kg}$ & $204 \mathrm{~kg}$ & $111 \mathrm{~kg}$ & $74 \mathrm{~kg}$ \\
\hline Basic power (no peak) & $620 \mathrm{~W}$ & $140 \mathrm{~W}$ & $175 \mathrm{~W}$ & $57 \mathrm{~W}$ \\
\hline $\mathrm{TM}$ rate & $0.9 \mathrm{Mbps}$ & $1.0 \mathrm{Mbps}$ & $1.4 \mathrm{Mbps}$ & $0.4 \mathrm{Mbps}$ \\
\hline
\end{tabular}

${ }^{1)}$ Effective area includes mirrors, detector efficiency and filter transmission

- The Wide-Field Imager (WFI) which has a mirror with a good HPD $(<15$ ") over a relatively large field-of-view, a moderate focal length $(2.75 \mathrm{~m})$, and a large cooled CCD detector.

- The Wide-Field Monitor (WFM, 2 units) is a coded mask instrument with a hard X-ray CdZnTe detector (8-200 keV) and a very large field-of-view (2.5 sr). It provides the capability to detect and localize GRBs and other transient events.

- The Gamma-Ray Burst Detector (GRBD 2 units) consists of crystal scintillators and extends the WFM energy range beyond $1 \mathrm{MeV}$. This provides extra triggering capability for GRB detection and is needed to determine the peak energy of GRBs with an accuracy of $10 \%$ or better. These detectors have no imaging capability.

For these instruments we will discuss the design and the current status in some detail. Their main characteristics are summarized in Table 3.

\subsection{Wide-Field Spectrometer (WFS)}

High resolution spectrometry is an essential capability of EDGE, and enables key measurements related to GRBs, the WHIM, and clusters. In order to meet the scientific requirements one needs the capability to disentangle weak emission and absorption features from prominent foreground emission and the instrumental background. Current advances in cryogenic detectors [10] with imaging capability and a spectral resolution of a few $\mathrm{eV}$ at $500 \mathrm{eV}$ make this feasible for the first time.

The instrument design includes a telescope with a conical shape with 2 and 4 fold reflections for the inner and outer shells respectively [11] and a TES array in a cryogen-free cooler, which will be cooled to $50 \mathrm{mK}$ (Fig. 8). After selecting a very short focal length (1.2 meter), the baseline FoV is $0.7 \times 0.7 \mathrm{deg}^{2}$ for a $32 \times 30$ array of 

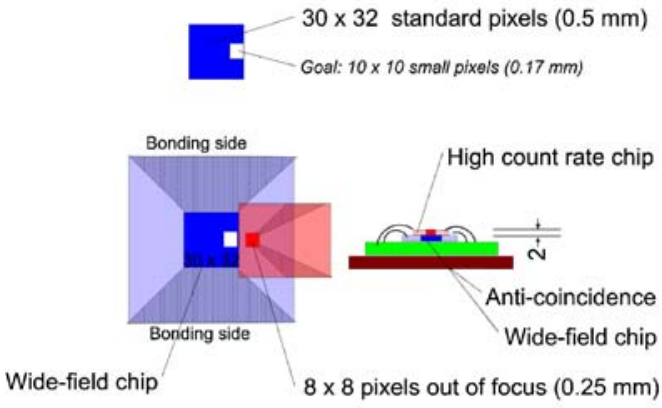

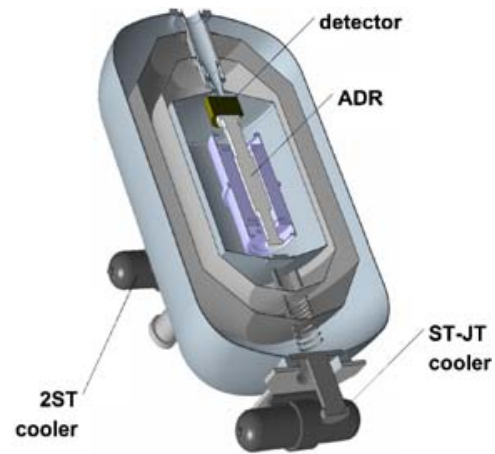

Fig. 8 Schematic design of the WFS detector proper (left) including the out-of-focus section to handle bright GRBs. At the right panel the cryogen free cooler is illustrated

$0.5 \times 0.5 \mathrm{~mm}^{2}$ pixels. The standard pixel has an energy resolution better than $3 \mathrm{eV}$, but smaller pixels with lower heat capacitance can achieve even better performance (a factor of 9 in heat capacitance translates into a factor of 3 in the energy resolution). Lithographic technologies allow for a hybrid device, of which a subset of the pixels has significantly better energy resolution. In addition, a small out-offocus section (64 pixels) can be included to avoid pile-up for observations of strong sources, like gamma-ray bursts in cases for which the count rate exceeds 200 counts/ $\mathrm{pixel} / \mathrm{s}$ (if the angular resolution is better than 4'). Operation at $50 \mathrm{mK}$ with $0.4 \mu \mathrm{W}$ cooling power can be obtained by a cryogenic-free cooler that requires $203 \mathrm{~kg}$ and $424 \mathrm{~W}$.

In Fig. 9 we show the energy resolution and GRASP of the WFS. An energy resolution of $<2 \mathrm{eV}$ has been demonstrated by the EURECA team on single pixels at the Bessy synchrotronfacilioty over an energy range between 0.15 and $2 \mathrm{keV}$ (the $\mathrm{Cu}$ absorber in these detectors needs to be replaced by a $\mathrm{Cu} / \mathrm{Bi}$ absorber to enlarge the pixels). A flight qualified cooler is being developed for the Japanese NeXT mission, due for launch around 2013. The principle of four-fold reflections has been verified. With a positioning error of the shells of $\pm 8 \mu \mathrm{m}$, already achieved on the Japanese Suzaku mission, the half power diameter is already 3.7'. The detection efficiency at
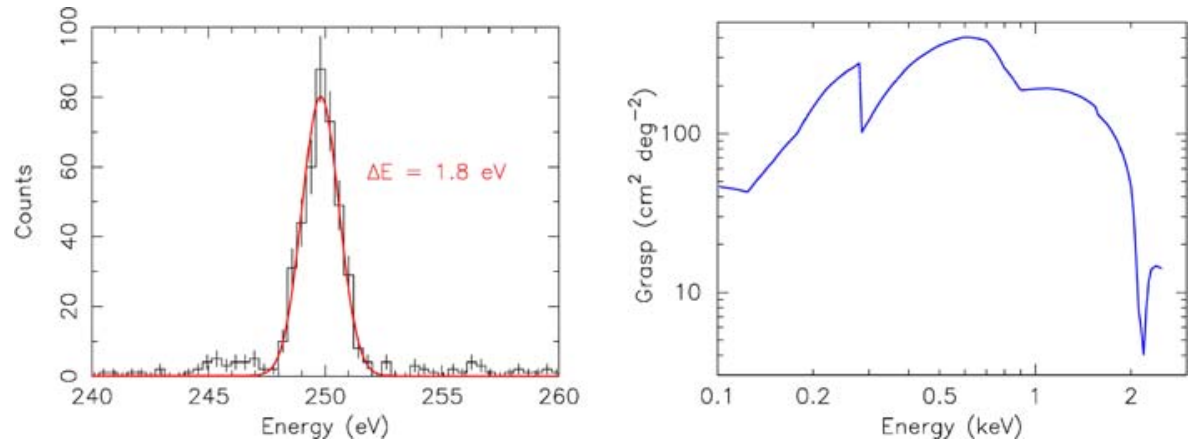

Fig. 9 Left: Measured energy resolution of the WFS detector at Bessy (tail to low energies is due to test setup aspects) and right: predicted grasp including mirror vignetting, detector filters and detector efficiency 
low energy is limited by four entrance filters $(50 \mathrm{~nm}$ parylene $+20 \mathrm{~nm} \mathrm{Al}$ on a $\mathrm{Si}$ support grid) at the different temperature stages of the cryostat. The mirror temperature is controlled using a long thermal baffle and $125 \mathrm{~W}$ heating power. No thermal blanket is required on the mirror to keep the mirror at its operational temperature.

\subsection{Wide-Field Imager (WFI)}

In order to detect point sources at a flux limit of $1.510^{-16} \mathrm{erg} \mathrm{cm}^{-2} \mathrm{~s}^{-1}(0.5-2 \mathrm{keV})$, required to measure accurately the outer regions of clusters and study the evolution of groups and clusters of galaxies from the formation epoch to the local Universe, the WFI must have a low background and good angular resolution. The low particle background of the MOS CCD technology is proven on XMM, and will be further reduced due to the Low Earth Orbit of EDGE. The cosmic X-ray background comprises a diffuse galactic component and an extragalactic component mostly due to unresolved sources. The angular resolution of the WFImager (HPD $<15$ ") will enable us to resolve the bulk of the latter component. The truly diffuse background and unresolved fraction of the extragalactic component will be characterized by long off-source observations and combining the high angular and high spectral resolution data.

Critical for the WFI is good angular resolution of the mirror $(<15$ ") and low scattering wings over the full field of view. These have been achieved using a polynomial approximation of the mirror shapes (5th order polynomial) and a radially tilted focal plane $\left(6^{\circ}\right)$. The use of $2 \mathrm{~mm}$ thick $\mathrm{SiC}$ mirror shells has been successfully demonstrated at different X-ray beam facilities [12]. We expect that a reduction to 1 $\mathrm{mm}$ thickness is a reasonable goal. In this case, while maintaining the good image quality, we will increase the effective area by $\sim 30 \%$ at low energies and $\sim 50 \%$ at high energies. In Fig. 10 we show the expected angular resolution including assembly errors.

To maintain the angular resolution over the full diameter of the field-of-view $\left(1.5^{\circ}\right)$ a mosaic of partially trapezoidal CCDs tilted by about $6^{\circ}$ is used. Thin CCDs, similar to the devices used in XMM-Newton, can easily be made in the required format and placed at tilted positions. The proposed tapered image section is of low
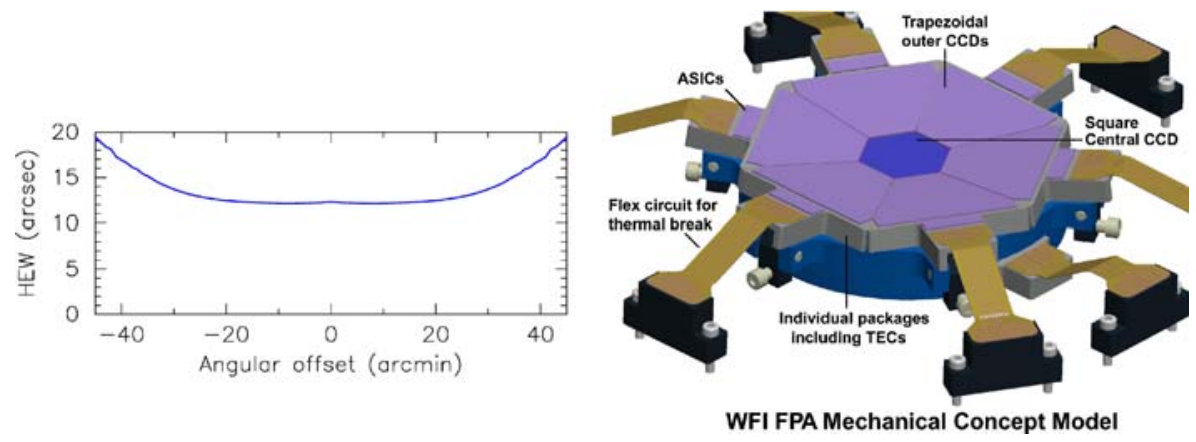

Fig. 10 Mirror PSF (HEW) as function of off-axis angle, including assembly errors (left) and the corresponding focal plane array employing a central rectangular CCD and 6 trapezoidal outer CCDs (tilted by $\left.6^{\circ}\right)$ 

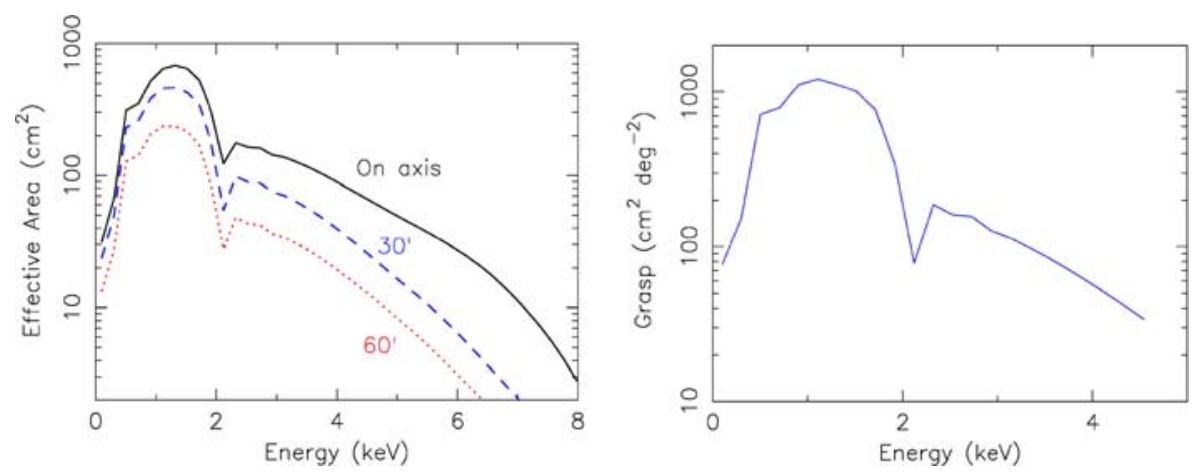

Fig. 11 Effective area of the WFI (including mirror, detector QE and thermal blanket) for different offaxis angles (left) and grasp (right) as function of energy

risk and has also been used on XMM-Newton (outside the field of view). The CCDs are passively cooled to $-50^{\circ} \mathrm{C}$, and a Thermal Electrical Cooler is used to further cool them to the operational temperature of $-80^{\circ} \mathrm{C}$. The telemetry is dominated by observations of bright Galactic sources and will handle sources up to $0.5 \mathrm{Crab}$ (50 bits/event). Following a GRB, the read-out of the 6 outer CCDs will be suspended, and the inner CCD will switch into a high count rate imaging mode (integration times of 0.1 and $2.5 \mathrm{~s}$ ) or into a windowed timing mode (1-D imaging) capable of counting 20,000 counts $\mathrm{s}^{-1}$ with acceptable pileup. Readout in these modes is followed by the standard 7-CCD photon counting mode as the GRB intensity decays. A filter wheel, operated at room temperature, will be incorporated to provide filter thicknesses optimized for different science programs. The filter wheel includes a calibration source for in-flight instrument calibration.

The predicted performance is shown in Fig. 11 which shows the effective area for a number of off-axis angles and the grasp, respectively. For the trapezoidal CCDs, the Quantum Efficiency (QE) is likely to vary by 5\% from low to high off-axis angle, which is $<10 \%$ of the mirror vignetting function. Both will require detailed modeling and calibration.

\subsection{Wide-Field Monitor (WFM)}

The Wide-Field Monitor [13] will monitor a $~ 3$ sr solid angle and will localize GRBs with a fluence greater than $10^{-6} \mathrm{erg} \mathrm{cm}^{-2}(15-150 \mathrm{keV})$, with a positional uncertainty better than 4'. It will locate a sufficient number of GRBs with afterglows bright enough to determine the absorption from the WHIM and measure the cosmic history of metals at GRB sites. However, lowering the threshold down to $5 \mathrm{keV}$ allows a substantial increase of X-ray flash (XRF) and high-Z GRB detections and this increases the number of bright afterglows useful for the WHIM and metallicity studies.

The design is based on two standard coded mask instruments with CdZnTe detectors with good efficiency between 8 and $200 \mathrm{keV}$ (2 mm thick). By tilting these instruments by $28^{\circ}$ with respect to the optical axis of the WFI and WFS, the sensitivity of the cameras can be optimized and is consistent with the 2.5 sr coverage (Fig. 12). For bursts as fluent as $210^{-6} \mathrm{erg} \mathrm{cm}^{-2}$ the FoV is even $3.7 \mathrm{sr}$. With a 
Fig. 12 Effective area of the WFM (2 units) indicating the limiting sensitivity for bursts at different fluences. The fluence limit corresponds to the required sensitivity

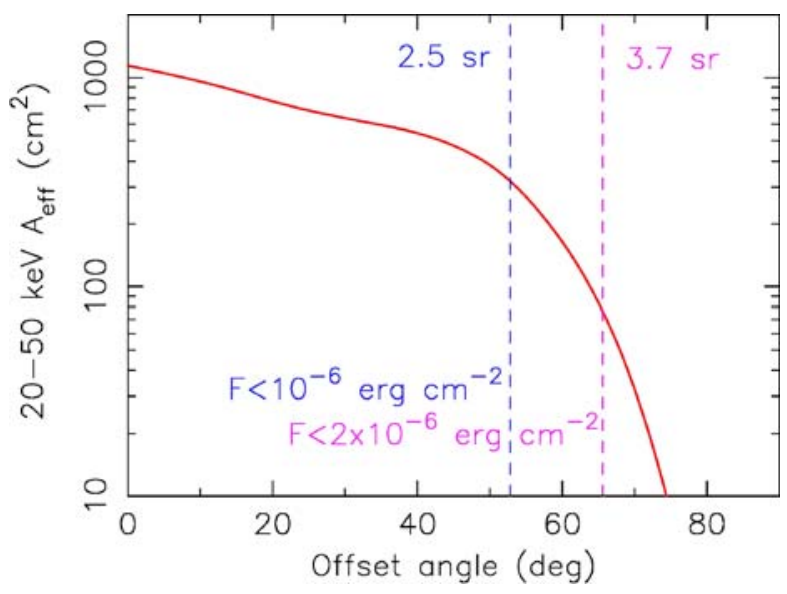

distance to the mask of $40.5 \mathrm{~cm}$ and a pixel size of $2.7 \mathrm{~mm}$, a location accuracy of 4' is achieved for sources detected with a $\mathrm{S} / \mathrm{N}>10$. This is sufficient to trigger fast repointing and to locate the source in the $6^{\prime} \times 6^{\prime}$ field-of-view of the high count rate section of the WFS. The on-board trigger will be based on sampling count rates with different integration times, and includes an image trigger based on an on-board catalogue of known sources. This procedure is standard in satellites like SWIFT [14] and AGILE [15]. Following the repointing, the data of the WFI can be used to tune the pointing to within $1^{\prime}$ (or better). The sensitivity of the camera is sufficient to detect and localize about 80 bursts with a prompt fluence of $>10^{-6} \mathrm{erg} \mathrm{cm}^{-2}$ per year and is comparable to the IBIS instrument.

\subsection{Gamma-Ray Burst Detector (GRBD)}

The prime goal of the GRB Detector (Fig. 13) is to measure the high-energy spectrum of GRBs, assessing their potential as standard candles for cosmology. Furthermore, we expect to use its results to avoid false triggers when we lower the WFM threshold to be more sensitive to high-z GRBs. For the measurement of the high-energy end of the spectrum we need to determine the peak energy $E_{p}$ with $\sim 10-20 \%$ accuracy for about
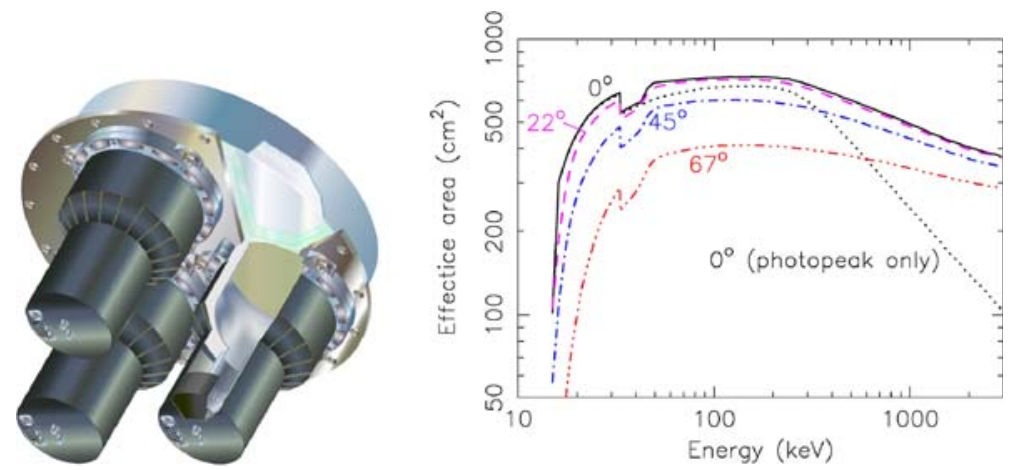

Fig. 13 Drawing of a GRBDetector and the related effective area of a single unit 
100-150 GRBs over the mission lifetime. This requires bandwidth extension to around $2 \mathrm{MeV}$, with sensitivity of about 2 photons $\mathrm{cm}^{-2} \mathrm{~s}^{-1}$ in the $50-300 \mathrm{keV}$ energy band, and co-alignment of this detector with the WFMonitor.

The design includes two scintillators (NaI, $850 \mathrm{~cm}^{2}$ each) read out by 3 PMTs. With a high ratio of photocathode area to scintillator area, the required energy resolution can easily be achieved. With an entrance window of about $0.5 \mathrm{~mm}$ thick Aluminum the lower threshold is $20 \mathrm{keV}$. Both units will be tilted by $45^{\circ}$ with respect to each other. Depending on the count rates, on-board spectra are generated with varying integration times ( $8 \mathrm{~s}$ and $16 \mathrm{~ms}$ during bursts), or raw events can be stored. The proposed detectors are similar to the GBM on GLAST [16].

\section{Spacecraft}

The spacecraft accommodation is shown in Fig. 14 and is mainly driven by the need to have a satellite with low mass and low moment of inertia, required for the fast repointing. Specific analysis was required for (a) the design of the attitude and orbit control system (AOCS), (b) the thermal design, and (c) the telecommunication. Other functions (e.g. data handling, electrical power) of the Service Module (SVM) have been designed using standard practice and will not be described in this paper.

\subsection{Attitude and Orbit Control System}

The AOCS design is driven by the required fast repointing capability and represents the most mission-specific capability of the SVM. The AOCS has been evaluated assuming two standard slews and one fast slew for each orbit. The trade off for the fast slew actuator selection has been performed considering the following possible solutions (a) reaction wheels (RW) only, (b) reaction wheels+thrusters, and (c) control moment gyro (CMG). The preliminary evaluation shows that the solution with Reaction Wheels only is the more viable solution. It is technically most mature (compared to $\mathrm{CMG}$ ) and its weight is acceptable (compared to RW+thrusters). The analysis has been performed for different off-the-shelf reaction wheels and these result typically in a slew time for $60^{\circ}$ of $60-65 \mathrm{~s}$ in $80 \%$ of the cases, $45-55 \mathrm{~s}$ for

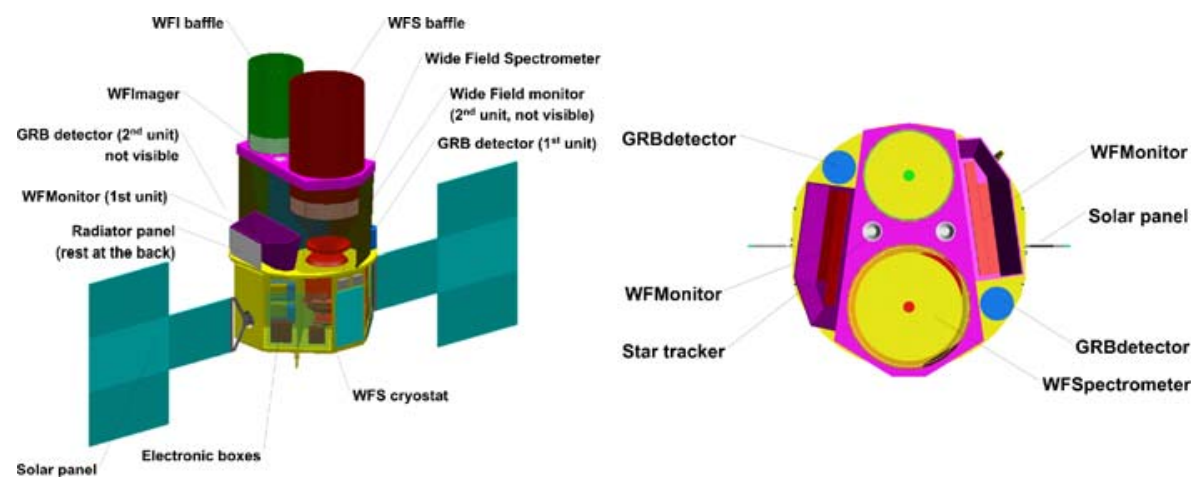

Fig. 14 EDGE payload accommodation (left: side view, right: top view) 
$50 \%$ and a maximum slew time of $85-90 \mathrm{~s}$. The required peak power for this approach $(2300 \mathrm{~W})$ can be accommodated by the power subsystem. The post-facto pointing accuracy can be as good as $<0.3-0.5^{\text {" }}$ ( 1 sigma) using a star tracker with a large FOV $\left(10^{\circ} \times 10^{\circ}\right.$ or $\left.20^{\circ} \times 20^{\circ}\right)$ and one high accuracy gyro which is based on offthe-shelf equipment.

\subsection{Thermal design}

The Thermal Control System (TCS) can be implemented following a standard approach: the radiators will be sized to reject the dissipative power in the hot case and the heating power will be sized to maintain the minimum equipment temperature requirement in the cold case. Typically we need about $4 \mathrm{~m}^{2}$ of radiative area (at the anti-sun side and the bottom of the satellite).

To keep the mirrors at the required temperature, despite the strong radiative cooling towards space we have adopted two different approaches. For the WFImager we have implemented a thermal blanket on top of the mirrors. This reduced the heating power for these mirrors to less than $40 \mathrm{~W}$ but reduces the low energy response (fortunately it also reduces the optical load of the detectors). For the WFSpectrometer, such reduction in the low energy response is not acceptable (the detector itself contains already thermal filters). In this case the temperature of the mirrors can be maintained by a combination of significantly more heating power $(125 \mathrm{~W})$, a significant longer optical/thermal baffle to reduce the viewing factor to deep space $(1 \mathrm{~m})$ and making use of less stringent thermal constraints on the mirror which has a angular resolution of only 3-4 arcmin.

Although a classical thermal design shows that a thermal design is feasible, considering the low earth orbit and the large power and cooling needs, this is clearly an area which can be significantly optimized by means of a detailed thermal and orbital analysis.

\subsection{Telecommunication}

This subsystem includes two main components: (a) A standard TM/TC link between $\mathrm{S} / \mathrm{C}$ and Earth for standard S/C operations. This standard link operates in S-band for a TC rate of about 2-4 kbps. For the TM rates we envisage the use of the high transmission rate $(3.8 \mathrm{Mbps})$, expected to be available for this mission and (b) A real time link for quick look alerts and telecommands will be used to allow GRB alerts to be rapidly transferred to the ground and to uplink target of opportunity positions. As a baseline we have identified the $S / C$-ORBCOMM low frequency link, already proven for the ASI-AGILE mission). Other solutions can be studied. For example a network of small receiving stations along the equator, like e.g. implemented in HETE2.

\section{Conclusions}

The EDGE mission will trace the cosmic history of baryons from early generations of massive star by Gamma-Ray Burst explosions through the period of cluster 
formation down to very low redshifts. The EDGE capabilities, in particular the wide field, high resolution spectroscopy, low background and fast reaction will open an unprecedented observational window on the core science but also on several other topics. The core science, which will distributed to the community directly, can be achieved in a 3 year mission still allowing for a reasonable guest observer program ( $20 \%$ of the observing time). The mission is unique and complementary to other existing and proposed missions and can be realized as an M-class mission within the ESA Cosmic Vision Program.

Acknowledgements The EDGE mission has been proposed to ESA as a medium class mission in the Cosmic Vision program. A large group of scientist from Europe, Japan and the USA has contributed to this proposal (see http://projects.iasf-roma.inaf.it/edge for the proposal proper). In addition the authors like to acknowledge the support of the Italian space agency (ASI) and the assistance of Thales Alenia Space for detailed technical assessment.

\section{References}

1. Piro, L., den Herder, J.-W., Ohashi T. et al.: EDGE proposal, arXiv:0707.4103v1 [astro-ph], available in high resolution at http://projects.iasf-roma.inaf.it/edge (2007)

2. Fukugita, M., Peebles, P.J.E.: ApJ. 616, 643 (2004)

3. Roncarelli, M., et al.: MNRAS. 373, 1339 (2006)

4. Vikhlinin, A., et al.: ApJ. 373, 1339 (2006)

5. Lumb, D.H., et al.: A\&A. 420, 853 (2004)

6. Borgani, S., Guzzo, L.: Nature. 409, 39 (2001)

7. Campana, S., et al.: A\&A. 449, 61 (2006)

8. Juett, A.M., et al.: ApJ. 648, 1066 (2006)

9. Takahashi, T. et al.: Proc SPIE, 6266, 6226-0D (2006)

10. de Korte, P.et al.: Proc SPIE, 6266, 6266-1Z (2006)

11. Sakurai, I. et al.: Proc SPIE, 668, pg 668809 (2007)

12. Citterio, O., et al.: Proc SPIE 3766, 198 (1999)

13. Natalucci, L. et al: Proc SPIE 6686, pg 6686-OT1 (2007)

14. Krimm, H.A.: Proc SPIE, 6266-04 (2006)

15. Tavani, M. et al.: Proc SPIE 6266-03 (2006)

16. Von Kienlin, A. et al.: Proc SPIE, 5488-125 (2004)

17. Borgani, S., et al.: MNRAS. 348, 1078 (2004)

18. Nicastro, F., et al.: Nature. 433, 495 (2005)

19. Rasmussen, A., et al.: ApJ. 656, 129 (2007)

20. Danforth, C.W., Shull, J.M.: ApJ. 624, 555 (2005)

21. Bromm V. Loeb A: to appear in "Gamma-ray Bursts" (CUP) arXiv:0706.2445v2 (2007) 